\title{
Bivariate spatiotemporal disease mapping of cancer of the breast and cervix uteri among Iranian women
}

\author{
Mehdi Raei, ${ }^{1}$ Volker Johann Schmid, ${ }^{2}$ Behzad Mahaki ${ }^{3,4}$ \\ ${ }^{1}$ Student Research Committee, School of Health, Isfahan University of Medical Sciences, Isfahan, Iran; \\ ${ }^{2}$ Bioimaging Group, Department of Statistics, Institue of Statistics, Ludwig Maximilans University, Munich, \\ Germany; ${ }^{3}$ Department of Epidemiology and Biostatistics, School of Health, Isfahan University of Medical \\ Sciences, Isfahan, Iran; ${ }^{4}$ Research Center for Environmental Determinants of Health, Kermanshah \\ University of Medical Sciences, Kermanshah, Iran
}

\begin{abstract}
Cervical cancer in women is one of the most common cancers and breast cancer has grown dramatically in recent years. The purpose of this study was to map the incidence of breast and cervix uteri cancer among Iranian women over a 6-year period (20042009) searching for trend changes and risk factors. Cancer incidence data were extracted from the annual reports of the National Cancer Registry in Iran. Hierarchical Bayesian models, including random spatial and temporal effects was utilized together with bivariate, spatio-temporal shared component modelling. The provinces Tehran, Isfahan, Mazandaran and Gilan were found to have the highest relative risk (RR) of breast cancer, while the highest RR of cervix uteri cancer was observed in Tehran, Golestan, Khuzestan and Khorasan Razavi. Shared risk factors (smoking component) between the two cancers were seen to have the highest influence in Tehran, Khorasan Razavi, Yazd, Isfahan, Golestan, Khuzestan, Fars and Mazandaran, while the least were observed in Kohgiluyeh Boyerahmad. Apparent differences and
\end{abstract}

Correspondence: Behzad Mahaki, Department of Epidemiology and Biostatistics, School of Health, Isfahan University of Medical Sciences, Hezar-Jerib Ave, Isfahan, Iran.

Tel.: 00989128077960 - Fax: 00983136682509.

E-mail: behzad.mahaki@gmail.com

Key words: Breast cancer; Cervix cancer; Bivariate disease mapping; Shared component model; Spatio-temporal analysis; Iran.

Contributions: MR, data entering; MR, BM analyzing; MR, manuscript writing; MR, BM, VJS, manuscript reviewing; BM, VJS study designing.

Conflict of interest: the authors declare no potential conflict of interest.

Funding: the work was supported by a grant [396547] from Isfahan University of Medical Sciences, Isfahan, Iran.

Received for publication: 9 November 2017.

Revision received: 7 March 2018.

Accepted for publication: 20 March 2018.

CCopyright M. Raei et al., 2018

Licensee PAGEPress, Italy

Geospatial Health 2018; 13:645

doi:10.4081/gh.2018.645 distinctions between high-risk and low-risk provinces reveal a pattern of obvious dispersion for these cancers in Iran that should be considered when allocating healthcare resources and services in different areas.

\section{Introduction}

Cancer is the third leading cause of mortality in Iran after cardiovascular disease and accidents (Mousavi et al., 2009). It is also considered one of the most important health problems in industrialized and developing countries (Mehrabani et al., 2008). While lung cancer is the most common cancer worldwide in both sexes, breast cancer is the most common malignancy in women (Cancer Facts \& Figures, 2016) and a major concern for general health in Iran (Montazeri et al., 2016). It accounts for about one third of all cancers in Iranian women and is the most common cause of death from cancer (Asgarian et al., 2016). While cancer of the cervix uteri has always been one of the most common cancers, breast cancer has grown dramatically during recent years (Poudel et al., 2016; Elidrissi Errahhali et al., 2017; Mathew et al., 2017). Although the prevalence of breast cancer in Iran is lower compared to many other Asian countries, the incidence among Iranian women has also increased (Mousavi et al., 2007; Taheri et al., 2012) so that the incidence per 100,000 women increased from 24 cases in 2006 to 28 in 2009. In addition, the average age when breast cancer is detected in Iran is 10 years younger than in other countries with a similar level of economic development (Pirouzpanah et al., 2014).

Gynaecologic cancers (cervical, ovarian, uterine, vaginal and vulvar) have profound effects on the various physical functions of women (Hasanzadeh Mofrad et al., 2015). Most of new cases of cervical cancer are diagnosed in middle-aged women with early stages of cancer (Le Borgne et al., 2013; Ramondetta, 2013), and metastases to the female genital tract from breast cancer are common (Manci et al., 2008). Different case studies have reported spread of breast cancer to the cervix (Ogino et al., 2003; Green et al., 2004) showing a prevalence ranging from $0.8 \%$ to $1.7 \%$ (Manci et al., 2008). Risk factors for breast cancer include stress, abortion, lack of lactation, positive family history, hormone therapy, low menstrual age, lack of delivery, alcohol intake, smoking and lack of physical activity (Toleutay et al., 2013; Jafarinia et al., 2016).

Cervical cancer is the second most common malignant neoplasm in women after breast cancer (Maree and Wright, 2011; Torre et al., 2015). Based on studies conducted in Iran, it is one of the five most common cancers among women with an incidence 
around 2.1 per 100,000 women per year (Farajzadegan et al., 2012; Chaichian et al., 2018). Cervix cancer is the third most common cancer among women in the United States (US), and the first or second among the cancers of the female genital system in many areas of Africa, India, Latin America, South and Southeast Asia (Vaisy et al., 2012). About $80 \%$ of women with this cancer live in the developing countries and its mortality rate is much higher in developing countries than in the industrialized part of the world (Morice and Castaigne, 2005). Smoking, having multiple sexual partners and use of contraceptive pills are potential risk factor for cervical cancer (Vaisy et al., 2012).

Increasing life expectancy has led to growth of the population aging index in Iran and it is expected that the incidence of various cancers in the coming years will increase accordingly (Asgarian et al., 2016). On the other hand, cancer distributions are subject to regional conditions, nutritional habits and genetic factors resulting in changes in the cancer incidence trends in different regions of Iran (Mahaki et al., 2011). For this reason it is essential to identify areas with low or high relative risk (RR) of cancer incidence, which can be served by obtaining accurate estimates of the incidence, prevalence and mortality rates of various forms of cancer from different parts of the country. Cancer mapping is far from uncommon in Iran (Mahaki et al., 2011; Asmarian et al., 2013; Jafari-Koshki et al., 2014; Chamanpara et al., 2015; HaddadKhoshkar et al., 2015; Khoshkar et al., 2015; Rastaghi et al., 2015), but spatiotemporal mapping of two or more cancers has not been attempted. It occurred to us that it would be of interest to apply spatio-temporal analysis of the variation of disease risk and carry out joint analysis of two cancer forms that share common environmental risk factors. Working on lung cancer, Richardson et al. (2006) used a Bayesian hierarchical model that splits the risk of disease into two spatio-temporal components, a shared and a specific component that calibrates the differential between the two diseases. In line with this thinking we applied a bivariate spatiotemporal shared component (SC) mapping of the incidence of two common cancers, those affecting breast and cervix uteri, and three risk factors associated with these cancers among Iranian women in the 30 provinces of Iran during a 6-year period (2004 to 2009). We aimed to study the dispersion of the RR of these two cancers using bivariate joint modelling, which has the ability to integrate correlations between geographically close regions and provide the opportunity to examine shared and specific patterns of the RR as well as temporal changes.

\section{Materials and Methods}

\section{Data}

We used information on two common cancers in women: that of the breast (topography code C50) and that of cervix uteri (C53) in women in the 30 provinces of Iran during 2004-2009. The data were collected from the annual reports of the National Cancer Registry in Iran, published by the Center for Disease Control of the Ministry of Health and Medical Education.

\section{Modelling}

Considering the bivariate spatial SC modelling method (Mahaki et al., 2011), the added temporal dimension to this model made it possible to study the temporal changes of the two cancers (breast and cervix cancer), including surrogate risk factors for the cancers (smoking, physical activity and use of contraceptive pills) were examined during a 6-year period from 2004 to 2009 using hierarchical Bayesian models, including random effects in place and time dimensions, using the equation:

$\mathrm{E}_{\mathrm{ijk}}=\frac{\sum_{\mathrm{i}} \mathrm{O}_{\mathrm{ijk}}}{\sum_{\mathrm{i}} \mathrm{N}_{\mathrm{ij}}} \cdot \mathbf{N}_{\mathrm{ij}}$

Eq. 1

where $O_{i j k}$ and $E_{i j k}$ are the numbers of observed and expected cases, respectively, of the $k^{\text {th }}$ cancer occurrence in the $i^{\text {th }}$ area and in $j^{\text {th }}$ time period. $E_{i j k}$ was obtained by multiplying the national incidence of the $k^{\text {th }}$ occurrence in the population of the $i^{\text {th }}$ province in the $j^{\text {th }}$ year $\left(\mathrm{N}_{\mathrm{ij}}\right)$. Spatio-temporal bivariate SC modelling was performed based on a hierarchical Bayesian modelling framework. Assuming that $O_{i j k}$ follows a Poisson distribution, i.e. $O_{i j k} \sim$ Poisson $\left(\Theta_{i j k} E_{i j k}\right)$ where $\theta_{i j k}$ is the unknown RR of the $k$ cancer in the $i^{\text {th }}$ area and in $j^{\text {th }}$ time period, the hidden factors (shared components) were introduced and the dispersion of the observed values modelled on $\theta_{i j k}$.

The simplest model is the one that only includes the cancerspecific intercept and shared spatio-temporal components (without heterogeneity and space-time interaction terms) along with the corresponding scale parameters written as:

$\log \left(\theta_{\mathrm{ij} 1}\right)=\alpha_{1}+\lambda_{1 \mathrm{i}} \cdot \delta+\lambda_{2 \mathrm{i}}+\mathrm{t}_{1 j} \cdot \psi+\mathrm{t}_{2 \mathrm{j}}$

$\log \left(\theta_{\mathrm{ij} 2}\right)=\alpha_{2}+\lambda_{1 \mathrm{i}} / \delta+\lambda_{3 \mathrm{i}}+\mathrm{t}_{1 \mathrm{j}} / \psi+\mathrm{t}_{3 \mathrm{j}}$

Eq. 2

where $\theta_{\mathrm{ij} 1}$ and $\theta_{\mathrm{ij} 2}$ are the RR of the first and second cancer, respectively, in the $i^{\text {th }}$ province in the $j^{\text {th }}$ time period, while represents the disease-specific intercept of the $k^{\text {th }}$ cancer; $\lambda_{1 \mathrm{i}}, \lambda_{2 \mathrm{i}}, \lambda_{3 \mathrm{i}}$ and $\mathrm{t}_{1 \mathrm{j}}, \mathrm{t}_{2 \mathrm{j}}, \mathrm{t}_{3 \mathrm{j}}$ are the spatial and temporal effects of the shared smoking component common to breast and cervix cancers, physical activity factors for breast cancer and use of contraceptive pills for cancer of the cervix uteri, respectively.

Spatial and temporal scale parameters $(\delta>0, \psi>0)$ indicate the weight of each shared component on the spatial and temporal changes of the cancers and can be different for each related cancer.

In total, there are four main models (A-D) that can be obtained by adding each space-time interaction effect $\left(\xi_{\mathrm{ij}}\right)$ and heterogeneity effects $\left(\varepsilon_{\mathrm{ij} 1}\right)$ to the original model. The models differ according to the inclusion or not of a heterogeneity term. In this way, a set of spatio-temporal models for bivariate mapping of diseases were introduced. The first model (A) was a simple additive decomposition of the shared part without heterogeneity and space-time interaction terms (Eq. 2). In the second model (B) we included $\varepsilon_{\mathrm{ij} 1}$ to capture possible variations not explained by the terms included in model $\mathrm{A}$. In model $\mathrm{C}$ we added space-time interaction terms common to all diseases $\left(\xi_{\mathrm{ij}}\right)$ to model A. Finally we built model D by combining models $\mathrm{B}$ and $\mathrm{C}$.

\section{Assigning prior distributions}

In this study, similar to the studies by Richardson et al. (2006) and Oleson et al. (2008), conditional autoregressive (CAR) prior distribution were used for spatial random effects $\left(\lambda_{1}\right)$. In this distribution, a unit weight is considered for neighbouring areas, and the neighbourhood matrix for this distribution is defined based on the conditions in adjacent areas. For time trends $\left(\mathrm{t}_{\mathrm{j}}\right)$, first order random-walk priors (Richardson et al., 2006; Oleson et al., 2008) were employed. In the present study, a simple interchangeable hierarchical structure was considered for $\xi_{\mathrm{ij}}$ (Knorr-Held and Besag, 1998). For heterogeneous expressions $\left(\varepsilon_{\mathrm{ij} 1}, \varepsilon_{\mathrm{ij} 1}\right)^{\mathrm{T}}$, multivariate normal distribution with zero mean, and the covariance matrix 
$\Sigma^{-1}$ was used in order to consider the correlation between the two diseases in each spatio-temporal unit.

For each $\alpha_{k}$, a flat non-informative prior was allocated and for spatial and temporal scales logarithms $(\log \delta$ and $\log \psi)$, it was assumed that they followed multivariate normal distributions. For precision parameters of spatial and temporal priors of the CAR model:

$$
\left(\tau_{\mathrm{t}}=\frac{1}{\sigma_{\mathrm{t}}^{2}}, \tau_{\lambda}=\frac{1}{\sigma_{\lambda}^{2}}\right)
$$

we acted according to the suggestions by Wakefield et al. (2001), which meant that both parameters were separately assigned the weekly informative gamma hyperprior distributions. For the precision matrix of the diseases-specific heterogeneity effects $\left(\Sigma^{-1}\right)$, the Wishart prior distribution $(\mathrm{Q}, 2)$ was employed where $\mathrm{Q}$ is the 2dimensional identity matrix. (Downing et al., 2008)

The suitability of all SC models to the data were assessed through Bayesian estimation software, WinBUGS 3.2.2 (Lunn et al., 2000). All maps were produced with ArcGIS 10.4.1 software (http://www.esri.com/software/arcgis). Posterior distribution estimations for model parameters were obtained by Monte Carlo Markov Chain simulation methods (Brooks, 1998). For each model, the Markov chain was executed with 100,000 iterations. The burn-in period was performed with 20,000 repetitions and results reported on the basis of the 80,000 remaining posterior samples with thin 10 to reduce the temporal autocorrelation (Spiegelhalter et al., 2002). Also, comparison of different spatiotemporal models was performed using the Deviance Information Criterion (DIC).

\section{Results}

The incidence of breast cancer in the 2004-2009 period was $3,803,4,621,5,378,6,181,8,145$ and 7,840, respectively. The incidence of cervix cancer during these 6 years was 394, 358, 436, 533, 630, and 572, respectively. Based on these results, despite slight fluctuations, the incidence of both cancers in the whole country showed an increasing trend.

Based on the occurrence or absence of spatio-temporal interaction effects and disease-specific heterogeneity effects, four main forms of the bivariate spatiotemporal SC model were investigated. Since the DIC index (used to compare the fitting of the four main models and selecting the optimal one) was only applied when the model with disease-specific heterogeneity effects without spatiotemporal interaction effects was preferable to other models (DIC=2241), only the results of this model is presented.

Figures 1 and 2 show the RR estimation maps based on the bivariate spatiotemporal SC model. According to the results with respect to breast cancer, the provinces Tehran, Isfahan, Mazandaran, Gilan, Khorasan Razavi and Yazd had the highest RR. However, the provinces Fars, Qazvin, Markazi, Qom, Semnan, Golestan, Kerman, Kermanshah and Bushehr provinces must also be considered high-risk areas but less so than the previous provinces mentioned. The provinces Tehran, Isfahan, Mazandaran and Gilan had the highest RR in each year investigated, while the provinces North Khorasan, Zanjan, Chaharmahal Bakhtiari, Sistan Baluchestan, and Kohgiluyeh Boyerahmad had the lowest (Table 1).

In the case of cancer of the cervix uteri, the provinces Tehran,
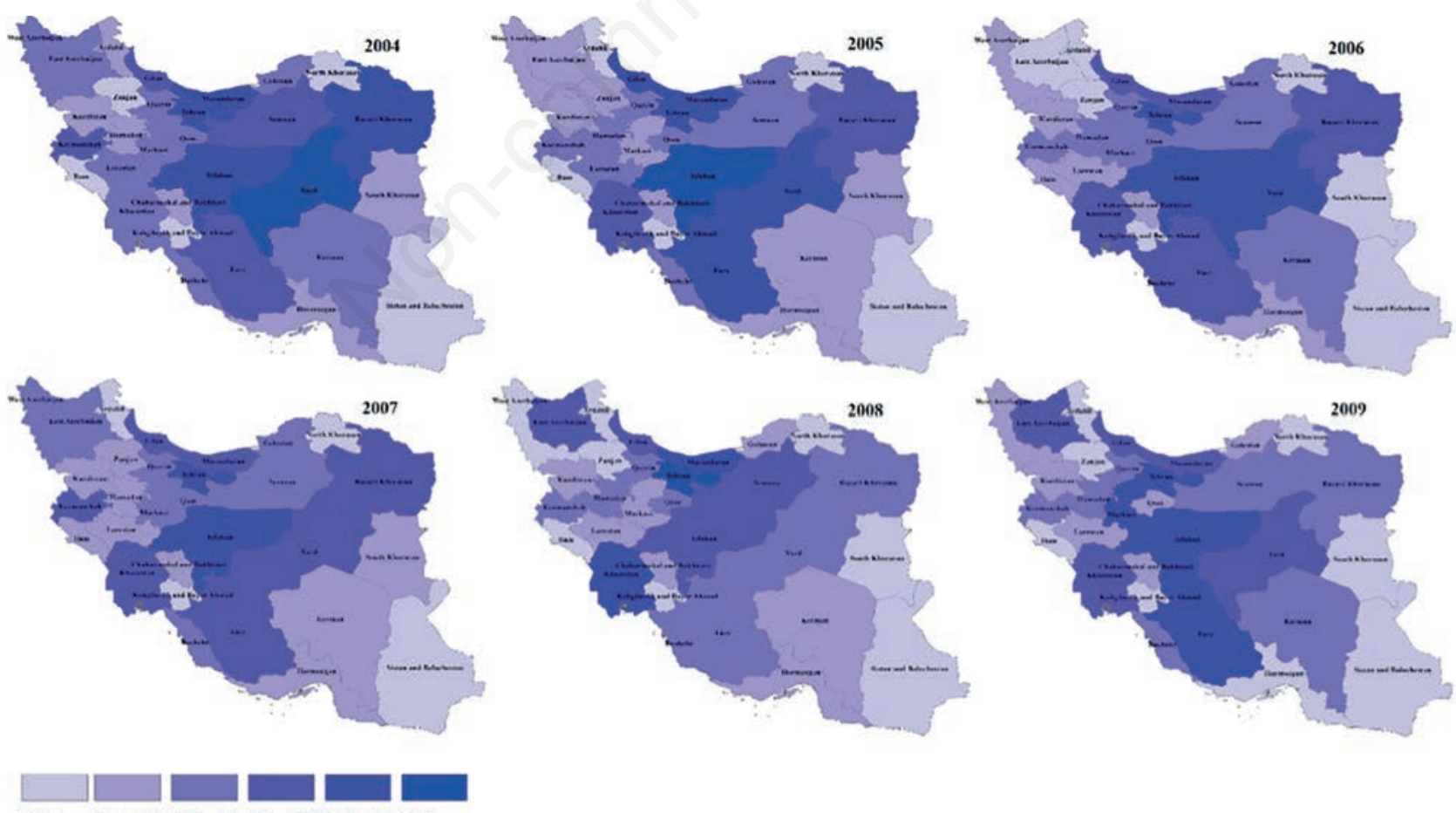

$<0.5 \quad 0.5 \cdot 0.75 \quad 0.75 \cdot 1.0 \quad 1.0 \cdot 1.251 .25 \cdot 1.5>1.5$

Figure 1. Distribution of the estimated relative risk due to breast cancer in Iran according to bivariate spatiotemporal shared component model in the period 2004-2009. 

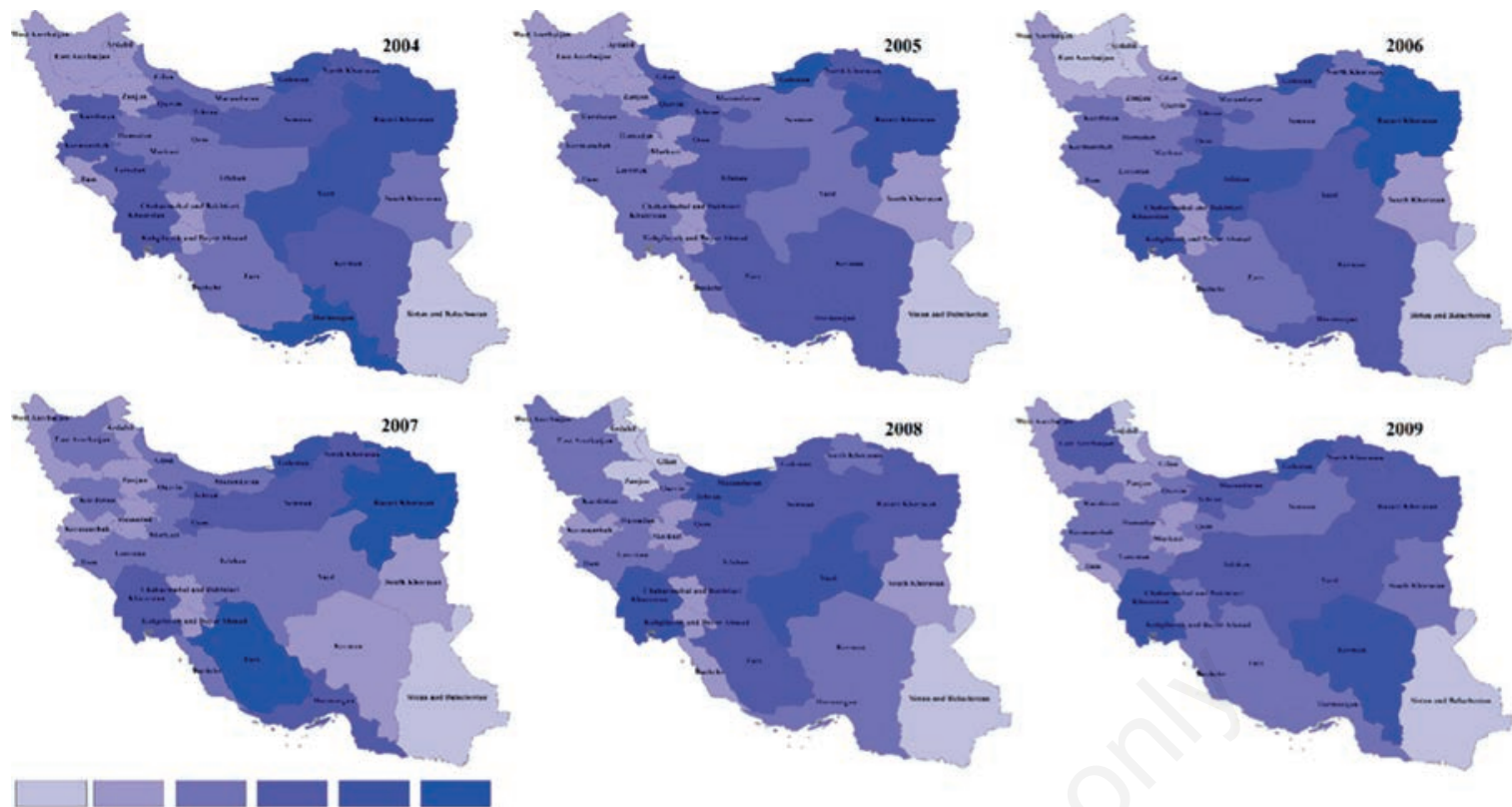

$<0.5 \quad 0.5-0.75 \quad 0.75 \cdot 1.0 \quad 1.0-1.25 \quad 1.25 \cdot 1.5>1.5$

Figure 2. Distribution of the estimated relative risk due to cervix uteri cancer in Iran according to bivariate spatiotemporal shared component model in the period 2004-2009.

Table 1. Relative risk of breast cancer in Iranian provinces.

\begin{tabular}{|c|c|c|c|c|c|c|}
\hline \multirow[t]{2}{*}{ Province } & \multicolumn{2}{|c|}{ High risk } & \multicolumn{2}{|c|}{ Some risk } & \multicolumn{2}{|c|}{ Low risk } \\
\hline & All years & Some years & All years & Some years & All years & Some years \\
\hline Ardabil & & & & & $\mathrm{x}$ & \\
\hline Bushehr & & & $\mathrm{X}$ & & & \\
\hline Chaharmahal Bakhtiari & & 10 & & & $\mathrm{X}$ & \\
\hline East Azerbaijan & & $\sqrt{2}+2$ & & $\mathrm{x}$ & & \\
\hline Fars & & 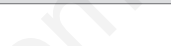 & $\mathrm{x}$ & & & \\
\hline Gilan & $\mathrm{x}$ & & & & & \\
\hline Golestan & & & $\mathrm{x}$ & & & \\
\hline Hamadan & & & $\mathrm{X}$ & & & \\
\hline Hormozgan & s & & $\mathrm{X}$ & & & \\
\hline Ilam & $\gamma_{1}$ & & & & & $\mathrm{X}$ \\
\hline Isfahan & $\mathrm{x}$ & & & & & \\
\hline Kerman & & & $\mathrm{x}$ & & & \\
\hline Kermanshah & & & $\mathrm{X}$ & & & \\
\hline Khorasan Razavi & & $\mathrm{x}$ & & & & \\
\hline Khorasan North & & & & & $\mathrm{X}$ & \\
\hline Khorasan South & & & & & & \\
\hline Kohgiluyeh Buyerahmad & & & & & $\mathrm{X}$ & \\
\hline Khuzestan & & $\mathrm{x}$ & & & & \\
\hline Kurdistan & & & $\mathrm{X}$ & & & \\
\hline Lorestan & & & & $\mathrm{X}$ & & \\
\hline Mazandaran & $\mathrm{x}$ & & & & & \\
\hline Markazi & & & $\mathrm{x}$ & & & \\
\hline Qom & & & $\mathrm{X}$ & & & \\
\hline Qazvin & & & $\mathrm{X}$ & & & \\
\hline Semnan & & & $\mathrm{X}$ & & & \\
\hline Sistan Baluchestan & & & & & $\mathrm{x}$ & \\
\hline Tehran & $\mathrm{X}$ & & & & & \\
\hline West Azerbaijan & & & $\mathrm{X}$ & & & \\
\hline Yazd & & $\mathrm{X}$ & & & & \\
\hline Zanjan & & & & & & $\mathrm{X}$ \\
\hline
\end{tabular}


Golestan, Khorasan Razavi, North Khorasan, Khuzestan and Kurdistan showed the higher RR level followed by the provinces Yazd, Hormozgan, Qom, Qazvin, Isfahan, and Kerman, which were also deemed to be at a higher risk than other provinces. The provinces Tehran, Golestan, khuzestan and Khorasan Razavi had the highest RR level in all six time periods, while the Zanjan, Chaharmahal Bakhtiari, Sistan Baluchestan, and Kohgiluyeh Boyerahmad provinces had the lowest (Table 2).

The estimated effects of the three shared spatial components $\left(\lambda_{\mathrm{i}}\right)$ can be seen in Figure 3. The component representing the effect of smoking on cancers of the breast and cervix uteri, had a higher effect in Tehran, Khorasan Razavi, Yazd, Isfahan, Golestan, Khuzestan, Fars and Mazandaran, respectively.

The use of contraceptive pills, considered to represent a specific risk for cervix uteri cancer, was fairly steady, but no pattern of association could be traced among the effects of this component across the provinces.

The pattern noticed regarding the component of low physical activity which was considered a specific risk with respect to breast cancer, on the other hand, was somewhat different. Although, this specific component had the highest effect in Gilan Province and the least in North Khorasan, there was no specific pattern of association between the effects of this component. In order to evaluate the temporal trend of changes in the effects of shared and specific component surrogate risk factors, the RR of these effects throughout the country was estimated and their results presented (Table 3 ). All RRs in each time period were near 1 and none was statistically significant. The RR weights of spatial and temporal effects were estimated to compare the role and importance of the surrogate components of risk factors in the spatial and temporal changes of the cancers studied. According to the results of spatial effects, the weight ratio of surrogates for shared risk factors of breast cancer to the weight of this component for cervix uteri cancer was 1.21 (95\%CI: 0.82-1.68). Therefore, the role of the surrogate for a shared risk factor in the geographical distribution of breast cancer incidence is greater than that of cervix uteri cancer, but this difference was not statistically significant. For temporal effects, the relative weight ratio was 0.95 (95\% CI: 0.48-2.1), which indicates that the effect of shared risk factors with respect to RR changes in breast and cervix cancers over time was not strongly different.

Table 2. Relative risk of cervix uteri cancer in Iranian provinces.

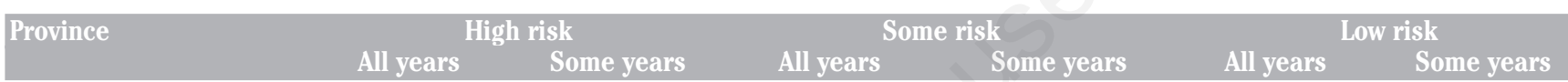

Ardabil

Bushehr

Chaharmahal Bakhtiari

East Azerbaijan

Fars

Gilan

Golestan

Hamadan

Hormozgan

Ilam

Isfahan

Kerman

Kermanshah

Khorasan Razavi

Khorasan North

Khorasan South

Kohgiluyeh Buyerahmad

Khuzestan

Kurdistan

Lorestan

Mazandaran

Markazi

Qom

Qazvin

Semnan

Sistan Baluchestan

Tehran

West Azerbaijan

Yazd

Zanjan

\section{All years} Some years x

Some years

All years

X

X

$\mathrm{x}$

X

X

X

X

X

\section{X}

$\mathrm{X}$

X X

$\mathrm{X}$

$\mathrm{X}$

$\mathrm{X}$

$\mathrm{X}$

$\mathrm{X}$ 


\section{Discussion}

This study aimed to determine the RR distribution of breast and cervix cancer in Iranian provinces as well as verifying the role of shared and specific risk factors for these cancers over time and place. The advantage of implementing the bivariate spatiotemporal $\mathrm{SC}$ model with latent variables (surrogates of risk factor) was that we could estimate the effect of shared and specific components of these factors without having the actual information on the risk factors themselves.

The number of provinces with some levels of RR for the incidence of these cancers was high. Therefore, we could detect a clear pattern of dispersion for them that can be used to reduce the imposed limits and costs by carefully planning of screening, early detection and treatment of these afflictions. Since the epidemiology of cancer in each region depends on various parameters, such as race, age, gender, cultural and social customs, nutritional status and individual living patterns (Alberg et al., 2005), it is necessary to examine the incidence patterns in the different regions.

The distribution of the RR of breast cancer in Iran during 2004-2009 provided in this research is corroborated by other studies (Norouzi Nejad et al., 2009; Mahaki et al., 2011; Lotfi et al., 2014). According to Iran's national cancer registry, breast cancer ranks first among cancers in Iranian women. Although the average age when breast cancer is detected is about ten years lower in Iran than in some other countries (Gohari et al., 2014), the high incidence level of breast cancer in Iran is not consistent with its decline in many other countries (Ferlay et al., 2010). New Western-style dietary habits, environmental pollution, increased smoking rate and population aging are indicated as some of the main reasons for the increase of cancer in the Iranian society (Fateh and Amini, 2008). Given the change in the pattern of risk factors and demographics, breast cancer is expected to increase dramatically in the coming years (Lotfi et al., 2014).

In this study, we found that the provinces Tehran, Golestan and Khorasan Razavi had the highest RR with regard to the incidence of cervix uteri cancer in all 6 years, which is consistent with the results of Baeradeh et al. (2015) and our results in other provinces were not unexpected.

The geographical pattern of breast and cervical cancers was also largely similar with the provinces Sistan Baluchestan, Kohgiluyeh Boyerahmad, Zanjan and Chaharmahal Bakhtiari at the least risk, and Tehran, Khuzestan, and Khorasan Razavi at the highest risk for both cancers. It is known that environmental and climatic conditions can be conducive to the incidence of cancer and its distribution. With the existing considerable geographical differences in Iran and the association of higher levels of cervical cancer with certain regions, it is more than probable that the risk of these cancers are largely determined by environmental factors. For example, vitamin D levels has a direct relation with the absorption of sunlight via the skin and is much more likely to occur in the southern areas (Heshmat et al., 2008). Indeed, factors such as living at northern latitudes, vitamin D deficiency and low sun exposure has been mentioned as conducive factors for the incidence of certain cancers in women, especially breast cancer (Grant, 2010;
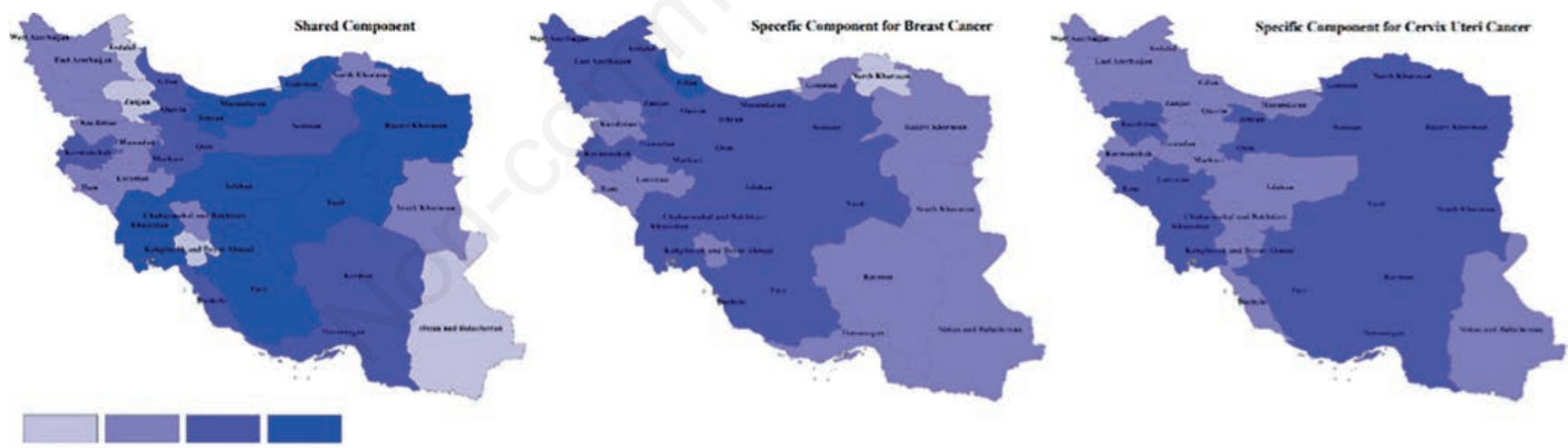

$<0.75$

$0.75 \cdot 1.01 .0 \cdot 1.25>1.25$

Figure 3. Map of the estimated relativa risk in Iran considering the spatial effect of shared and specific components based on the bivariate spatio-temporal shared component model.

Table 3. The estimated relative risk of temporal effects of shared and specific components.

\begin{tabular}{|c|c|c|c|c|c|c|}
\hline \multirow[t]{2}{*}{ Type of component } & \multicolumn{6}{|c|}{ Posterior median (\%95 confidence interval) per year } \\
\hline & 2004 & 2005 & 2006 & 2007 & 2008 & 2009 \\
\hline Shared component & $\begin{array}{c}1.01 \\
(0.96-1.08)\end{array}$ & $\begin{array}{c}1.00 \\
(0.96-1.05)\end{array}$ & $\begin{array}{c}0.99 \\
(0.95-1.03)\end{array}$ & $\begin{array}{c}0.99 \\
(0.95-1.03)\end{array}$ & $\begin{array}{c}0.98 \\
(0.93-1.02)\end{array}$ & $\begin{array}{c}0.99 \\
(0.94-1.05)\end{array}$ \\
\hline Specific components for breast cancer & $\begin{array}{c}1.01 \\
(0.95-1.07)\end{array}$ & $\begin{array}{c}1.00 \\
(0.95-1.05)\end{array}$ & $\begin{array}{c}1.00 \\
(0.96-1.04)\end{array}$ & $\begin{array}{c}0.99 \\
(0.96-1.04)\end{array}$ & $\begin{array}{c}0.98 \\
(0.93-1.03)\end{array}$ & $\begin{array}{c}0.99 \\
(0.93-1.05)\end{array}$ \\
\hline Specific components for cervix cancer & $\begin{array}{c}1.01 \\
(0.94-1.10)\end{array}$ & $\begin{array}{c}1.00 \\
(0.94-1.07)\end{array}$ & $\begin{array}{c}0.99 \\
(0.93-1.04)\end{array}$ & $\begin{array}{c}0.99 \\
(0.93-1.04)\end{array}$ & $\begin{array}{c}0.99 \\
(0.93-1.05)\end{array}$ & $\begin{array}{c}0.99 \\
(0.92-1.07)\end{array}$ \\
\hline
\end{tabular}


Walentowicz-Sadłecka et al., 2013; Lotfi et al., 2014).

The spatial map of surrogates for shared risk factors of the two cancers found is, however, uneven, with very little difference between the effects of breast cancer-specific surrogates. Also, the spatial map of surrogates for specific risk factors of cervix cancer was fairly smooth, and all provinces had moderate RRs. This component showed the least changes compared to other risk factors. The differences in the incidence of cancer in the different geographical areas are likely to vary due to the dominant risk factors. For example, diet and nutritional status, as well as the existence of different ethnic groups, can be considered as shared or specific factors for these cancers in northern Iran (Grumet and Bruner, 2000; Rafiei et al., 2012).

The temporal effects of the risk factor surrogates were almost constant and did not show any significant difference. This is predictable due to the relatively short duration of the study period and reveals the necessity of studies covering longer time periods.

\section{Conclusions}

The results of this study can guide Iranian health planners and policymakers in preventing the disease based on specific geographical, cultural and social characteristics of the country. We hope that the results of mapping the RR of breast and cancer of the cervix uteri using new spatio-temporal models to be considered by Iranian health planners and authorities when allocating resources and services to the health sector, taking geographical differences into account. Also, since most cancer patients do not have medical care insurance coverage, better access to cancer care should be a top priority for health policies in Iran.

\section{References}

Alberg AJ, Brock MV, Samet JM, 2005. Epidemiology of lung cancer: looking to the future. J Clin Oncol 23:3175-85.

Asgarian F, Mirzaei M, Asgarian S, Jazayeri M, 2016. Epidemiology of breast cancer and the age distribution of patients over a period of ten years. Iran J Breast Dis 9:31-6.

Asmarian NS, Ruzitalab A, Amir K, Masoud S, Mahaki B, 2013. Area-to-area poisson kriging analysis of mapping of countylevel esophageal cancer incidence rates in Iran. Asian Pac J Cancer Prev 14:11-3.

Baeradeh N, Mirzae M, Zamani M, 2015. Epidemiology of prevalent cancers in Khorasan Razavi province in 2008. MJMS 57:926-31.

Brooks SP, 1998. Markov chain Monte Carlo method and its application. J R Stat Soc Ser D-Stat 47:69-100.

Cancer Facts \& Figures, 2016. Worldwide data. Available from: https://www.wcrf.org/int/cancer-facts-figures/worldwide-data.

Chaichian S, Khateri S, Moradi Y, Khosravi Shadmani F, Mansori K, Khazaei Z, Moradpour F, Varse F, 2018. Trends in cervical cancer incidence in Iran from 2003 to 2009. MEJC 9:57-63.

Chamanpara P, Moghimbeigi A, Faradmal J, Poorolajal J, 2015. Joint disease mapping of two digestive cancers in Golestan Province, Iran using a shared component model. Osong Public Health Res Perspect 6:205-10.

Downing A, Forman D, Gilthorpe MS, Edwards KL, Manda SO, 2008. Joint disease mapping using six cancers in the Yorkshire region of England. Int J Health Geogr 7:41.

Elidrissi Errahhali M, Elidrissi Errahhali M, Ouarzane M, Boulouiz R, Bellaoui M, 2017. Cancer incidence in eastern Morocco: cancer patterns and incidence trends, 2005-2012. BMC Cancer 17:587.

Farajzadegan Z, Nourbakhsh S, Mostajeran M, Loghmani A, 2012. Cervical cancer screening status in 35 to 60 year-old women in Isfahan, Iran. J Isfahan Med Sch 30:1542-8.

Fateh S, Amini M, 2008. An epidemiologic study of colorectal cancer in Arak during 1994-2004. Iranian J Surg 2:11-7.

Ferlay J, Shin HR, Bray F, Forman D, Mathers C, Parkin DM, 2010. Estimates of worldwide burden of cancer in 2008: GLOBOCAN 2008. Int J Cancer 127:2893-917.

Gohari MR, Mokhtari Hesari P, Moghaddami Fard Z, Khoda Bakhshi R, 2014. Determining pattern of metastasis and prognostic factors in breast cancer using conditional regression model (PWP). SJSPH 12:41-52.

Grant WB, 2010. An ecological study of cancer incidence and mortality rates in France with respect to latitude, an index for vitamin D production. Dermatoendocrinol 2:62-7.

Green AE, Biscotti C, Michener C, Belinson J, 2004. Isolated cervical metastasis of breast cancer: a case report and review of the literature. Gynecol Oncol 95:267-9.

Grumet SC, Bruner DW, 2000. The identification and screening of men at high risk for developing prostate cancer. Urol Nurs 20:15-8.

Haddad-Khoshkar A, Jafari-Koshki T, Mahaki B, 2015. Investigating the incidence of prostate cancer in Iran 20052008 using Bayesian spatial ecological regression models. Asian Pac J Cancer Prev 16:5917-21.

Hasanzadeh Mofrad M, Karami Dehkordi A, Mozaffar Tizabi N, Amirian M, 2015. Survey of sexual dysfunction in women with cervical cancer and a history of pelvic radiation therapy in 2009 to 2013 in Ghaem and Omid hospitals, Mashhad. IJOGI 18:9-18.

Heshmat R, Mohammad K, Majdzadeh S, Forouzanfar M, Bahrami A, Ranjbar Omrani G, 2008. Vitamin D deficiency in Iran: A multi-center study among different urban areas. Iran J Public Health 37.

Jafari-Koshki T, Schmid VJ, Mahaki B, 2014. Trends of breast cancer incidence in Iran during 2004-2008: A Bayesian spacetime model. Asian Pac J Cancer Prev 15:1557-61.

Jafarinia B, Bahadorzai M, Delpisheh A, Sayehmiri K, Tavakoli M, 2016. Risk factors of breast cancer in Dezful city of Iran: a case-control study. Tehran Univ Med J 74:135-9.

Khoshkar AH, Koshki TJ, Mahaki B, 2015. Comparison of bayesian spatial ecological regression models for investigating the incidence of breast cancer in Iran, 2005. Asian Pac J Cancer Prev 16:5669-73.

Knorr-Held L, Besag J, 1998. Modelling risk from a disease in time and space. Stat Med 17:2045-60.

Le Borgne G, Mercier M, Woronoff A-S, Guizard A-V, Abeilard E, Caravati-Jouvenceaux A, Klein D, Velten M, Joly F, 2013. Quality of life in long-term cervical cancer survivors: a population-based study. Gynecol Oncol 129:222-8.

Lotfi B, Esmaeil Nasab N, Aghaei A, Ahmadi Jouybari T, 2014. Evaluation of breast cancer in Iranian women in an ecological study based on latitude, sun exposure and vitamin D intake. IJOGI 17:17-25.

Lunn D, Thomas A, Best N, Spiegelhalter D, 2000. WinBUGS-a Bayesian modelling framework: concepts, structure, and 
extensibility. Stat Comput 10:325-37.

Mahaki B, Mehrabi Y, Kavousi A, Akbari M, Waldhoer T, Schmid V, Yaseri M, 2011. Multivariate Disease Mapping of Seven Prevalent Cancers in Iran using a Shared Component Model. Asian Pac J Cancer Prev 12:2353-8.

Manci N, Marchetti C, Esposito F, Graziano M, Tomao F, Pastore M, Bellati F, Panici PB, 2008. Late breast cancer recurrence to the uterine cervix with a review of the literature. Int J Gynecol Pathol 27:113-7.

Maree J, Wright S, 2011. Cervical cancer: does our message promote screening? A pilot study in a South African context. Eur J Oncol Nurs 15:118-23.

Mathew A, Sara George P, M CK, G P, K MJ, Sebastian P, 2017. Cancer incidence and mortality: district cancer registry, Trivandrum, South India. Asian Pac J Cancer Prev 18:1485-91.

Mehrabani D, Tabei S, Heydari S, Shamsina S, Shokrpour N, Amini M, Masoumi S, Julaee H, Farahmand M, Manafi A, 2008. Cancer occurrence in Fars Province, Southern Iran. Iran Red Crescent Med J 10:314-22.

Montazeri V, Jafarpour Sadeghi F, Hosseinpour S, Mirzaei H, Akbari A, Ehsani M, Akbari S, Asadi N, Mahmoudinezhad M, Mirtaheri E, Sanaat Z, Pirouzpanah S, 2016. Reproductive risk factors of breast cancer among women in Tehran and Northwest of Iran: A case-control study. IRJE 12:1-9.

Morice P, Castaigne D, 2005. Advances in the surgical management of invasive cervical cancer. Curr Opin Obstet Gynecol 17:5-12.

Mousavi S, Gouya M, Ramazani R, Davanlou M, Hajsadeghi N, Seddighi Z, 2009. Cancer incidence and mortality in Iran. Ann Oncol 20:556-63.

Mousavi S, Montazeri A, Mohagheghi M, Mousavi Jarrahi A, Harirchi I, Najafi M, Ebrahimi M, 2007. Breast cancer in Iran: an epidemiological review. Breast J 13:383-91.

Norouzi Nejad F, Ramezani Daryasar R, Ghafari F, 2009. Epidemiology of cancer in Mazandaran province 2006. J Mazandaran Univ Med Sci 19:61-5.

Ogino A, Nomizu T, Gonnda K, Okouchi C, Sakuma T, Yamada M, Katagata N, Watanabe F, Yamaguchi Y, Yoshida T, 2003. A case of breast cancer metastasizing to cervix after resection of pancreatic metastasis. Breast Cancer 10:284-8.

Oleson J, Smith B, Kim H, 2008. Joint spatio-temporal modeling of low incidence cancers sharing common risk factors. JDS 6:105-23.
Pirouzpanah S, Taleban F, Mehdipour P, Atri M, Hooshyareh-Rad A, Sabour S, 2014. The biomarker-based validity of a food frequency questionnaire to assess the intake status of folate, pyridoxine and cobalamin among Iranian primary breast cancer patients. Eur J Clin Nutr 68:316-23.

Poudel KK, Huang Z, Neupane PR, 2016. Trend of Cancer Incidence in Nepal from 2003 to 2012. Asian Pac J Cancer Prev 17:2171-5.

Rafiei M, Akbari ME, Alizadeh M, Eshrati B, Hatami H, 2012. Geographical distribution and survival rate of cancers among elderly iranians. Payesh 11:603-9.

Ramondetta L, 2013. What is the appropriate approach to treating women with incurable cervical cancer? J Natl Compr Canc Netw 11:348-55.

Rastaghi S, Jafari-Koshki T, Mahaki B, 2015. Application of Bayesian multilevel space-time models to study relative risk of esophageal cancer in Iran 2005-2007 at a county level. Asian Pac J Cancer Prev 16:5787-92.

Richardson S, Abellan JJ, Best N, 2006. Bayesian spatio-temporal analysis of joint patterns of male and female lung cancer risks in Yorkshire (UK). Stat Methods Med Res 15:385-407.

Spiegelhalter D, Best N, Carlin B, Linde AVD, 2002. Bayesian measures of model complexity and fit (with discussion). J Royal Stat Soc 64:583-639.

Taheri NS, Bakhshandehnosrat S, Tabiei MN, Kashani E, Rajaei S, Besharat S, Semnani S, Roshandel G, 2012. Epidemiological pattern of breast cancer in Iranian women: is there an ethnic disparity? Asian Pac J Cancer Prev 13:4517-20.

Toleutay U, Reznik V, Kalmatayeva Z, Smigelskas K, 2013. Risk factors of breast cancer in kyzylorda oblast of Kazakhstan: a casecontrol study. Asian Pac J Cancer Prev 14:5961-4.

Torre LA, Bray F, Siegel RL, Ferlay J, Lortet-Tieulent J, Jemal A, 2015. Global cancer statistics, 2012. CA Cancer J Clin 65:87108.

Vaisy A, Lotfinejad S, Zhian F, 2012. Relationship between utrine cervical carcinoma and oral contraceptives. J Gorgan Uni Med Sci 14:98-103.

Wakefield J, Best N, Waller L, 2001. Bayesian approaches to disease mapping. Spatial Epidemiology 1:104-28.

Walentowicz-Sadłecka M, Sadłecki P, Walentowicz P, Grabiec M, 2013. The role of vitamin $D$ in the carcinogenesis of breast and ovarian cancer. Ginekol Pol 84:305-8. 